\title{
Beitrag zur Erforschung der funktionellen Bedeutung der Gewebsmastzellen.
}

\author{
Von \\ Yosio Kitayama. \\ (Mit ro Abbildungen und 4 Tabellen). \\ Aus dem Anatomischen Institut der Medizinischen Universität zu Kanažawa. \\ (Vorstand: Prof. Dr. K. Okamoto.)
}

\section{Fragestellung.}

Was die physiologische Funktion und Bedeutung der Gewebsmastzellen (im folgenden verkürzt ,,Mz.") anlangt, steht man recht verschiedenen Ansichten gegenüber, die sich je nach der Auffassung der chemischen Beschaffenheit der Zellgranula von einander unterscheiden.

- Einige betonten, daß die $\mathrm{Mz}$ : mit dem Ernährungszustand des Gewebes in innigem Zusammenhang stehen (Ehrlich, Westphal, Maximow, Pappenheim, Nagoyo-Nakajima) und andere waren der Meinungen, $\mathrm{da}$ sie an der Bildung von Bindegewebsfasem (Staemmlet), elastischer Substanz (Löwenthal, Josef), Fett-Zellen (Heitzmann), Schleim (Hoyer, Arnold, Harris) oder Nervenfasem (Korybutt-Daszkiewiecz) Anteil nehmen.

Auf Grund der Färbungsmethoden mit Rongalitweiss, Benzidin, und Wasserstoffperoxyd hatten wieder andere (Unnä, Golodetz, Miura) in den Mz.-Körnern Träger einer Peroxydase nachweisen wollen und betont, daß sie für die Oxydation des Gewebes irgend eine wichtige Rolle spielen sollten. Anderseits wurde die Anschauung vertreten, man könne in den Mz. mehr oder weniger degenerierte Zellformen sehen; z.B. Raudnitz bezeichnete sie als mucinös degenerierte Zellen, Polja k off als atrophische Fettzellen, Flèmming als Leichen von Bindegewebszellen.

Außerdem sollten die $\mathbf{M z}$.' sekretorische Elemente, Speichelzellen; phagozytäre Elemente, Puffer der Gewebs-pH (Miura), Vorstufe der Chromatophoren oder Träger des Lipoides sein. Jedoch erheben sich diese oben erwähnten Ansichten über die biologische Bedeutung der Mz. meist 
nicht über bloße Vermutungen, weil die bisherige Forschungsarbeit keine entsprechenden Anhaltspunkte für die Aufklärung der chemischen Zusammensetzung der Mz.-Granula herbeigebracht hat. •

Seit einigen Jahren werden tiefschürfende Untersuchungen über die Verteilung und Bedeutung der chromotropen Substanzen im Tierorganismus von Holmgren u.a. ausgeführt. Infolgedessen sind Chemismus und Funktion der Mz. etwas klarer geworden und wird von dieser Seite als neue Ansicht darüber behauptet, daß die metachromatischen Granula der Mz. mit dem Heparin (nach Jorpes ist es chemisch ein Mucoitinpolyschwefelsäureester) identisch seien und daß das weitverzweigte Zellensystem, welches die Ehrlichschen Mz. bildet, als die Bildungs- und Sekretions-Stätte eines antikoagulierenden Stoffes angesehen werden könne. Diese Ansicht gründet sich darauf, daß der Esterschwefelgehalt verschiedener Organe parallel mit dem Vorkommen von Mz. und mit dem Reichtum an metachromatischer Substanz geht. Z.B. ist die Kapsel der Kuhleber speziell reich an Mz., welche reichlich metachromatische Granula enthalten und ihr Esterschwefelgehalt ist außerdem ungewöhnlich hoch (0.066\%), während man aus Organen, welche besonders spärlich Mz. enthalten (wie Milz des. Kaninchens oder der Ratte), nur Spuren von Esterschwefelsäure erhielt.

Diese Autoren stellten auch aus der Kapsel der Kuhleber und der Pferdleber eine Substanz her, welche eine deutliche Heparinwirkung zeigt und auch dies steht mit der von ihnen vertretenen Ansicht in Einklang durch die Tatsache, daß die Mz.mit Vorliebe um den Gefäßwänden der Organe und Gewebe vorkommen.

Zwar haben Holmgren und Wilander in der Abhandlung ,Beitrag zur Chemie und Funktion der Ehrlichen Mz." (1937) die chemische Natur der Granula geklärt und dadurch neues Licht in des Chaos des Mz.-problems gebracht, doch scheint es mir, als ob hinsichtlich der funktionellen Bedeutung der betreffender Zellen, noch einige Fragen ungelöst geblieben seien.

Wenn die metachromatischen Granula, wie sie betonten, die Hemmung der Koagulation des fliessenden Blutes in Gefäße hervorrufen soll, so müssen die $\mathrm{Mz}$. in irgend einer Weise gegen die Bedingungen, welche die Gerinnugszeit des Blutes stark zu beschleunigen vermögen, reagieren. Es fehlt aber in ihrer Untersuchung der Versuch eine Entscheidung über diese logischerweise vorauszusetzende Funktion der Mz. herbeizuführen und deshalb kann auch ihre Schlußfolgerung über die funktionellen Bedeutung der $\mathrm{Mz}$. sich nicht über eine bloBe Vermutung erheben. Unter diesem Gesichtspunkt werden in der vorliegenden Arbeit die Ergebnisse einiger Untersuchungen über das Vethalten der Zellart gegenüber einer künstlich hervorgerufenen, bedeutenden Abkürzung der Koagulationszeit des Blutes-dargelegt. 


\section{Untersuchungsmaterial und Methode.}

Als Untersuchungsmaterialien wurden bei diesem Versuche Kaninchen, Meerschweinchen und Ratten verwendet, welche alle ganz gesund und mäßig gut emährt waren.

Die einfachste Methode, die Blutgerinnungszeit abzukürzen ist bekanntlich die unmittelbare Einführung von einem Blutkoagulans in die Gefäße der Versuchstiere. Also wurde hier auch die Injektionsmethode gebraucht und dadurch war es möglich den erwarteten Zustand künstlich zuwege zubringen. Als Injektionsmittel verwendete ich "Thrombogen Fuji“", welches nach dem Resultat der Vorprüfung die Blutgerinnungszeit bis unter ein Drittel des normalen Wertes abkürzen konnte. A

Tabelle $\mathrm{I}$.

\begin{tabular}{|c|c|c|c|c|c|}
\hline $\begin{array}{l}\text { Laufende } \\
\text { Nummer }\end{array}$ & Tierart & $\begin{array}{l}\text { Körper- } \\
\text { gewicht } \\
\text { (in gr.) }\end{array}$ & $\begin{array}{c}\text { Tagesdosis } \\
\text { des } \\
\text { injizierten } \\
\text { Thrombogen }\end{array}$ & Injektionsorte & $\begin{array}{c}\text { Zeitdauer } \\
\text { der } \\
\text { Injektion }\end{array}$ \\
\hline$x$ & Ratte & .65 & 0.2 c.c. & intraperitoneal' & 5 Tage \\
\hline 2 & $n$ & 60 & 0.2 c.c. & . n & $7 n$ \\
\hline 3 & $\pi$ & 63 & 0.5 c.c. & subcutan & $5 \pi$ \\
\hline 4 & n & ss & 0.5 c.c. & $n$ & $7 n$ \\
\hline 5 & ${ }^{n}$ (Kontrast) & 60 & - & - & - \\
\hline 6 & Meerschweinchen & 580 & x.6 c.c. & intraperitoneal & 7 Tage \\
\hline 7 & $n$ & 600 & r.5 c.c. & subcutan & 10 \\
\hline 8 & (Kontrast) & 550 & $\longrightarrow$ & : & \\
\hline 9 & $\left(\begin{array}{l}n \\
n\end{array}\right)$ & 570 & $\longrightarrow$ & $\longrightarrow$ & , \\
\hline 10 & Kaninchen & 1380 & 2.0 c.c. & Ohrvenen & 7 Tage \\
\hline II & $n$ & 1450 & $2.0 \mathrm{C.c}$ & $n$ & Io \\
\hline 12 & (Kontrast) & 1420 & - & - & $\longrightarrow$ \\
\hline
\end{tabular}

Wie aus Tabelle I hervorgeht, habe ich das Mittel bei Kaninchen direkt in die Randvenen des Ohres injiziert, doch bei Meerschweinchen und Ratte wurden Bauchhöhle und subcutanes Gewebeder Rückenhaụt als die Injektionsorte gewählt. Die injizierte Tagesdosis sowie auch die Zeitdauer der Injektion sind aus obiger Tabelle ersichtlich. .Vor dem Experimente habe ich die Gerinnungszeit des Blutes von jedem Tiere mehrmals sorgfältig gemessen und ihre durchschnittlichen Normalwerte vorausgehend festgestellt. 
Die Injektion wurde täglich zu bestimmter Zeit ausgeführt und zwei Stunden danach entnahm ich den Tieren Blut, um die Veränderung der Gerinnungszeit zu messen. Das Blut wurde bei Kaninchen aus den Randvenen des Ohres, bei Meerschweinchen durch Herzpunktion direkt aus dem Herz oder durch Stich aus der größten Ohrvene und bei Ratte durch Stich oder Abschneiden des Schwanzes aus der Schwanzvene entnommen.

Beim Studium der Messung der Blutgerinnungszeit war es möglich, die - von In ou je und Sas a ki neu entworfene einfache Messungsapparatur durch die Liebenswürdigkeit Herm Prof. Dr. In ouje zu verwenden.

Ich möchte hier nicht versäumen, Herrn Prof. Dr. In ouje für seine Liebenswürdigkeit, meinen aufrichtigsten Dank auszusprechen.

Tabelle $\dot{2}$.

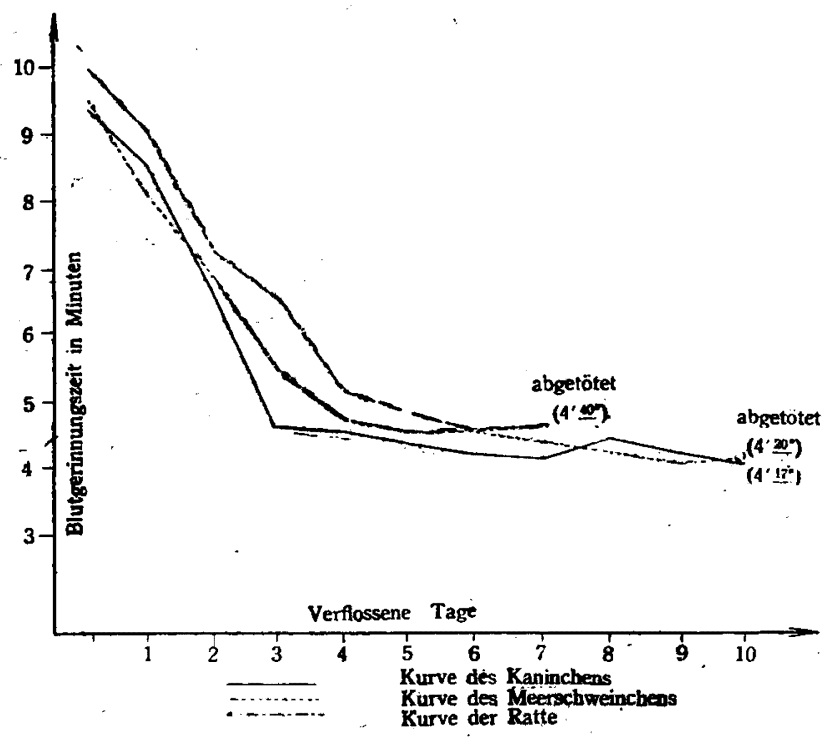

In der Tabelle 2 ist die Blutgerinnungszeit jedes Tieres vor der Experiment und die Veränderung derselben nach der Injektion in Kurven dargestellt.

Der Normalwert der Blutgerinnungszeit jedes Tieres beträgt bei Kaninchen durchschnittlich 9 Minuten und 20 Sekunden, bei Meerschweinchen 9 Minuten und 28 Sekunden im Mittel und bei Ratte 9 Minuten und $4^{8}$ Sekunden im Mittel. Diese Werte werden allmählich kürzer, wie man aus den Kurven ablesen kann, fallen bis unter die Hälfte vom Anfangswert in 4 Tagen und erreichen nach 5-7 Tagen einen minimalen Grenzwert. (abtöten.)

Alle Tiere wurden gerade nach der letzten Messung der Blutgerinnungszeit mittels Chloroform abgetötet und obduziert. Die Organe und Gewebe, 
Tabelle 3.

\begin{tabular}{|c|c|c|c|c|c|c|}
\hline \multirow{2}{*}{ Organe } & \multicolumn{2}{|c|}{ Ratte } & \multicolumn{2}{|c|}{ Meerschweinchen } & \multicolumn{2}{|c|}{ Kaninchen } \\
\hline & Experim. & Kontrast & Experim. & Kontrast, & Experim. & Kontrast \\
\hline Zunge & 4 & $x$ & 2 & 2 & 2 & I \\
\hline Oesophagus & 2 & I & 2 & 2 & 2 & I \\
\hline Magen - & 4 & $\mathbf{r}$ & 2 & 2 & 2 & I \\
\hline Dünndarm & 4 & $\mathbf{I}$ & 2 & 2 & 2 & I \\
\hline Leber & 4 & $\mathbf{I}$ & 2 & 2 & 2 & $\mathbf{I}$ \\
\hline Trachea & - & - & - & - & 2 & $\mathbf{I}^{\prime}$ \\
\hline Lunge & 4 & $\mathrm{I}$ & 2 & 2 & 2 & I \\
\hline Niere & 4 & I & 2 & 2 & 2 & $\mathbf{I}$ \\
\hline Ureter & - & - & 2 & $\dot{2}$ & - & - \\
\hline Harnblase & - & - & 2 & 2 & 2 & I \\
\hline Herz & 2 & $\mathbf{I}$. & 2 & 2 & 2 & I \\
\hline große Gefäße & 4 & I & 2 & 2 & 2 & I \\
\hline Milz & 4 & $\mathbf{r}$ & 2 & 2 & 2 & I \\
\hline Gehirn & 2 & I & 2 & 2 & 2 & I \\
\hline Periphere Nerven & - & - & -2 & 2 & - & - \\
\hline Pancreas & 4 & $\mathbf{I}$ & 2 & 2 & 2 & I \\
\hline Nebenniere & 4 & $I$ & 2 & 2 & 2. & $\mathbf{I}$ \\
\hline $\begin{array}{l}\text { Hoden, Nebenhoden } \\
\text { u. Samenleiter }\end{array}$ & 2 & - & I & $x$ & 2 & I \\
\hline $\begin{array}{c}\text { Ovarium, Uterus u. } \\
\text { Eileiter }\end{array}$ & 2 & $\mathbf{I}$ & I & I & - & - \\
\hline Muskel & 4 & r & -2 & 2 & 2 & I \\
\hline Haut & 4 & $\mathbf{r}$ & 2 & 2 & 2 & I \\
\hline
\end{tabular}

welche ich genauer Untersuchung unterzogen habe, sind in Tabelle 3 angegeben.

Bei der Herstellung der mikroskopischen Präparaten aus diesen Organen bediente ich mich der vorig von mir mitgeteilten Methode zur Fixierung und Färbung der Gewebsmazstellen. Kleine Stücke der verschiedenen Organen wurden in die I \%ige Trypaflavinlösung 24 Stunden lang gelegt und dann im $60 \%$ igen Alkohol übertragen. Entwässerung und Praffineinbettung iwurden wie in üblicher Weise ausgeführt. Die Blocke wurden in $5 \mu$ dicke Serien- 
schnitte abgeschnitten, worunter ein Schnitt für je fünf Serien aufgenommen und nach der Entparaffinierung in Xylor ohne nennenswertige Ausführung 'sofort ins Canadabalsam eingeschlossen wurde. Außerdem verwendete ich die verbesserte Holmgrens Methode, welche die basische Bleiazetdt-Lösung als Fixationsmittel benutzt.

\section{Beschreibung der Befunde.}

\section{Zunge.}

Kaninchen: In den Epithezellen der Zungen-Schleimhaut der kontrastierten Tiere sowie auch der injizierten Tiere findet man keine Mz., jedoch kommen bei den Präparaten der Kontrastreihe spärliche rundliche oder ovale Mz. mit mäßig reichlichen mittel großen Granula in dem Unterschleimhautgewebe und in den Zungenmuskeln vor. Sie liegen haupisächlich entlang des Gefäße einführenden Bindegewebes oder dicht an den Kapillarenwänden.

Bei den Präparaten der Experimentreihe ist es schwer eine Vermehrung der Mz.-Zahl nachzuweisen, doch findet man deutliche Abnahme und Aufquellung der Zellgranula, welche etwas blässer gefärbt sind. Die Form der Zelle ändert sich, sie wird mehr oder minder langgestreckt oder spindelig.

Meerschweichen: Bei Präparaten der Kontrastreihe gibt es mehrere rundliche, ovale oder spindelige mit reichliche mittelgroße Granula versehene Mz. in der Submucosa und der Zungenmuskulatur. Bei den Tieren der Injektionsreihe zeigen sich die $\mathrm{Mz}$. etwas vermehrt in der Kapillarenwand der betreffenden Organe. Die durchschnittliche Zahl der Mz. in je io Gesichtsfeldern der ganzen Präparate beträgt bei Kontrastreihen ca. 5 und bei Injektionsteihen $8 \mathrm{im}$ Mittel in der gleichen 130 fachen Vergrößerung (im vorliegenden wird die Zahl der Mz. unter den hier erwähnten Bedingungen angegeben). Die Zellgranula nehmen auch ansehnlich an $\mathrm{Zahl}$ ab und sind etwas aufgequollen. Die Formen der Zellen werden mehr oder weniger langgestreckt oder polygonal.

Ratte: Die Befunde der Präparate der Kontrastreihe sind fast gleich den Befunden des Kaninchens. Bei Tieren der Injektionsreihe findet man nur spärliche ovale oder langgestreckte $\mathrm{Mz}$. mit recht verringerten groben Granula in den betreffenden Organen.

\section{Speiseröhre.}

Kaninchen: Bei der kontrastierte Reihe findet man fast keine oder eventuell nur einige kleine rundliche ovale oder etwas langgestreckte $\mathrm{Mz}$. mit 
mäßig reichlichen mittelgroßen Granula in dem Unterschleimhautbindegewebe. Durch die Experimentreihe kann man weder Vermehrung der Mz. noch Veränderung der Zellformen in den Präparaten nachweisen, jedoch ist die Färbbarkeit der Granula ansehnlich herabgesetzt und ihre Volumina etwas aufgequollen.

Meerschweinchen: Es gibt keine Mz. in Mucosa, Submucosa und Muskelhaut der Speiseröhre, sondern nurspärlich in der Faserhaut und dem den Oesophagus umgebenden Bindegewebe ebensowohl bei kontrastierten als auch injizierten Präparaten. Vermehrung der Mz.-Zahl ist nicht sicher konstatierbar, aber die Form der Zelle ändert sich, sie ist etwas langgestreckt. Die Zellgranula sind aufgequollen, ihre Menge verringert.

Ratte: Nur einige Mz: werden in Submucosa und dem Bindegewebe der Muskelhaut der koritrastierten Speiseröhre aufgefunden. Sie sind rundlich oval oder spindelig und mit mäßig reichlichen Granula versehen. Ihre Zell-Granula sind fast in gleicher Größe mit denen des Kaninchens und werden tiefgefärbt. Die Befunde der Experimentreihen ähneln den Befunden beim Kaninchen. Nur die Veränderungen an den Zellgranula sind positiv nachweisbar geworden.

\section{Magen.}

Die Stücke wurden hauptsächlich aus dem Corpusteile entnommen.

Kaninchen: Bei den Tieren'der Kontrastreihe befinden sich nur einige rundliche kleine $\mathrm{Mz}$. mit geringeren Granula im Bindegewebe der Tunica propria der Magenschleimhaut. Weder in Submucosa noch in Stratum musculare kann eine einzige $\mathrm{Mz}$. nachweisen. Bei den Tieren der Injektionsreihe findet man am Körper- oder Basalteile der Zotten der Magenschleimhaut ca: 8, in der Submucosa oder Muskélschichte nur einige oval oder langgestreckte Mz. in der Gefäßwandgegend. Die Granula sind abgeblasst, verringert sowie etwas aufgequollen.

Meerschweinchen: In der Tunica propria, der Submucosa und der Muskelhaut der kontrastierten Reihen befinden sich mehrere (ca- 6 im Mittel) kleine, den Lymphozyten ähnliche Mz., welche mit mäßig reichlichen Zellgranula versehen, sind. Bei den Experimentreihen beträgt die Mz. Zahl in Mucosa, Submucosa und Muskelhaut durchschnittlich 12 in einem Gesichtsfeld. Sie zeigen hier wie in anderen Organen das ubiquitäre Vorkommen in der Gefäßwandung. Die Formen der Zellen sind oval, langgestreckt oder polygonal und die Menge der Zellgranula hat erheblich abgenommen.

Ratte : Zahl und Form der Mz. in der Kontrastreihe sowie auch in der Experimentreihe sind nicht abweichend von den bei Kaninchen beschriebenen 
Mz. Die Menge der Zellgranula nimmt nach der wiederholten Injektion ansehnlich ab, während ihre Volumina etwas aufquellen.

\section{Dünndarm.}

Kaninchen : Bei der Kontrasteihe sind gar keine Mz: in den Präparaten auffindbar, jedoch bei der Experimentalreihe treten einige kleine rundliche oder ovale granulaarme $\mathrm{Mz}$. in der Tunica propria der Darmschleimhaut auf. Die Größe der Zellgranula hat etwas zugenommen, aber ihre Menge ist stark reduziert.

Meerschweinchen: In Mucosa, Submucosa und Muskelschicht der kontrastierten Organe findet man relativ reichliche (ca. is Zellen im Mittel) rúndliche oder ovale, selten langgestreckte Mz., welche mäßig reichliche Zellgranula enthalten. Bei der Experimentreihe nimmt die Zahl der Mz. ansehnlich zu (27 Zellen im Mittel), doch die Menge der Granula'ab.

Ratte: An der Präparaten beider Reihen kann man fast keine $\mathrm{Mz}$. nachweisen. Nur in einigen Präparaten aus injizierten Tieren findet man einzelne kleine rundliche granulaarme Mz. in der Tunica propria oder der Submucosa der Darmschleimhaut.

\section{Leber.}

Kaninchen: Bei Tieren der Kontrastreihe findet man gar keine oder nur spärliche Mz. im interlobulären Bindegewebe oder entlang des Zentralvenen einführenden Bindegewebes. Sie sind klein, oval oder rundlich und mit schwach gefärbten geringeren Granula versehen. Bei Tieren der Experimentreihe zeigen sich als Ganzes mehrere kleinovale oder spindelige granulaarme Mz. im Leberparenchym oder im interlobulären Bindegewebe. Die Menge der Zellgrąnula nimmt ansehnlich ab.

Meerschweinchen: Bei den Kontrastreihen sind durchschnittlich $6 \mathrm{Mz}$. um die Zentralvenen oder im Parenchym nachweisbar. Die Zellen sind meist mittelğroß und oval, spindlig oder quadratförmig, deren Granula sind mäßig reichlich und gleich gross. Bei den Experimentreihen beträgt die Zahl der Mz. ca. 9 im Mittel, liegen meist um die Zenltralvenen und um die Gefäße der Glissonschen Kapsel. Die Form der Zellen sind rundlich, oval, spindlig oder langgestreckt. Die Menge der Granula hat abgenommen und ihr Färbbarkeit ist etwas herabgesetzt. (Abb. I u. 2.)

Ratte : In'der Leber des kontrastierten Tieres findet man gar keine Mz., aber im Parenchym der Leber aus der Experimentreihe kann man nur einige ovale Mz. mit geringeren Granula in ganzen Präparatserien nachweisen. 


\section{Trachea.}

Der Luftröhrenbefund wird nur bei einem Kaninchen im Versuche festgestellt. Bei Präpataten der Kontrastreihe gibt es keine Mz. weder in Mucosa, Submucosa noch in Muskelschicht der Trachea. Nur einige befinden sich in dem die Trachea umgegebenen Bindegewebe, welche spindlig oder langoval, mit mäßig reichlichen Granula versehen sind. Bei Präparaten der Experimentreihe kann man keine nennenswerte Veränderung der Mz. nachweisen.

\section{Lùnge.}

Kaninchen: Bei Präparaten der Kontrastreihe findet man mehrere (ca. 8 Zellen) ovale, spindlige oder langgestreckte granulaarme Mz., in der Pleura visceralis und dern subpleuralen Gewebe. Im Parenchym der Lunge bea finden sich nur einige in einem ganzen Präparat. Dagegen treten sie in Präparaten der Experimentreihe reichlicher auf in der Pleura und dem subpleuralem Gewebe (ro Zëllen im Mittel). Sie kommen vereinzelt auch im Lungenparençhym vor, etwas häufiger längs der Gefäße und den Broñchen. Die Formen der Zellen sind oval, langgestreckt oder sternförmig und die Granulamenge hat etwas abgenommen.

Meerschweinchen; in der kontrastierten Lunge des Meerschweinchens kommen die Mz. recht reichlich vor. Sie liegen meist in der Pleura und im subpleuralem Gewebe, worin die Mz.-Zahl durchschnittlich 2 I beträgt. Die Zellen sind relativ groß und vielgestaltig, enthalten mäßig reichliche Granula. Bei der injizierten Tieren findet man durchschnittlich 35 Zellen in einem Gesichtsfeld, welche mit Vorlieb in der Pleura visceralis und dem subpleuralem Gewebe vorkommen. Die Form der Zellen und die Menge der Granula weichen nicht deutlich von denen in der kontrastierten Lunge beschriebenen ab. Einige $\mathrm{Mz}$. befinden sich im Lungenparenchym sowie in der Gefäßwandung und im Bindegewebe der Bronchen. (Abb. 3, 4, 5, 6).

Ratte: Die Befunde der Rattenlunge sind fast gleich den Befunden, welche bei der Kaninchenlunge geschildert sind.

\section{Niere.}

Kaninchen: Es gibt in ganzen Präparaten nur einige kleine rundliche oder ovale $\mathrm{Mz}$. mit geringeren Granula in der Nierentinde der kontrastietten Tiere. Sie liegen teils an der Bowmannschen Kapsel teils an dem Tubulus contortus. Bei Nieren der In jektionsteihe kann eine Vermehrung der Mz.Zahl, eine Veränderung der Zellformen und die Abnahme der Zellgranula nicht mit Sicherheit konstatiert werden. 
Meerschweinchen: Mehrere (ca. s) Mz. treten in der Pars intermedia der kontrastierten Niére auf. Sie sind meist langgestreckt und enthalten mäßig reichliche Granula. Der Befund der Niere aus der Experimentreihe weicht nicht deutlich von dem in den Kontrastpräparaten ab.

Ratte: Sowohl in der kontrastierten Niere als auchin der Niere aus der Injektionsreihe findet man nur einzelne rundliche granulaarme $\mathrm{Mz}$. in der Nähe der Oberfläche der Nierenrinde.

\section{Ureter.}

Meerschweinchen : In den kontrastierten Präparaten kommen mehrere langgestreckte granulareiche $\mathrm{Mz}$. in der Tunica propria sowie in-der Glattenmuskelschichte vor. Sie treten auch vereinzelt um die Gefäße in der Faserhaut des Harnleiters auf. Bei den injizierten Tieren findet man durchschnittlich is Mz., welche langgestreckt, stemförmig oder mit langen Ausläufern versehen sind und an den oben erwähnten Stellen des Harnleiters liegen. Die' Zellgranula haben erheblich abgenommen und ihre Volumina sind aufgequollen.

\section{Harnblase.}

Kaninchen : In der Epithelschicht der kontrastierten Hamblase befinden sich keine Mz., aber in der Tunica propria sowie in der Glattenmuskelschicht findet man mehrere (ca: 7 Zellen im Mittel) kleine rundliche, ovale oder langgestreckte granulaarme Mz:, besonders um die Gefäße in der Submucosa und der Muskelhaut. Bèi Präparaten der Injektionsreihe kommen durchschnittlich $12 \mathrm{Mz}$. vor, welche meist langgestreckt sind und nur verringerte aufgequollene Granula um die Kerne enthalten.

Meerschweinchen: Bei den Präparaten der Kontrastreihe findet man etwa s Mz., welche oval, dreieckig oder langgestreckt und mit recht reichlichen Granula versehen sind: Hier kann auch der innige Zusammenhang der Mz. mit den Blutgefäßen nicht abgesprochen werden. Bei den Präparaten der Injektionsreihe befinden sich durchschnittlich ca: ${ }_{4} \mathrm{Mz}$. um die Gefäße in der Submucosa und der glatten Muskelschichte der Harnblase. Die Menge der Zellgranula nimmt ab und ihre Volumina sind aufgequollen.

\section{Herz und Großgefäße.}

Bei allen Versuchstieren werden als Großgefäß= Aorta abdominalis und Vena cava caudalis verwendet.

Kaninchen: Bei der Kontrastreihe werden keine Mz. weder in Herz noch in der unteren Hohlvene und Bauchaorta gefunden. Nur im Herz- 
muskel der Tiere aus der Versuchsteibe findet man einige ovale granulaarme $\mathrm{Mz}$. in der Nähe der Gefäßwandung.

Meerschweinchen: Man kann techt reichliche Mz. (ca. 20 im Mittel) in der Media bzw. in der Adventitia der Bauchaorta und unteren Hohlvene nachweisen. Sie sind klein Oval spindelig oder quadratförmig und mäßig granulareich. In dem Epicardium findet man durchschnittlich $27 \mathrm{Mz}$., fast dieselben Zellen, wie die in der großen Gefäßen beschriebenen. In Präparaten der Versuchsreihe beträgt die durchschnittliche Zahl der Mz. 35 in dem Epicardium, 26 in Bauchaorta und 29 in Vena cava caudalis. Die Formen der Zellen sind meist langgestreckt oder polygonal und enthalten geringere Mengen von Granula: (Abb. 7 7. 8).

Ratte: Die Befunde der Ratten zeigen fast keinen Unterschied gègenüber der Befunden beim Kaninchen.

\section{Milz.}

Kaninchen: In der Milz der kontrastierten Tieren findet man fast keine Mz., nur in einigen Präparaten treten einzelne rundliche granulaarme Mz. in der Pulpa auf. Bei der Versuchsteihe kann man höchstens bis zu 3 $\mathrm{Mz}$. in ganzen Präparatserien finden, welche auch rundlich oder oval und granulaarm sind.

Meerschweinchen : In den ganzen Präparaten der Kontrastreihe findet man nur vereinzelte Mz. in der Kapsel und dem Trabekel. Bei Tieren aus der Versuchsreihe befinden sich durchschnittlich $3 \mathrm{Mz}$., welche oval, spindelig oder dreieckig und mit blaß gefärbten Granula versehen sind, im Präparat aus dem Trabekel und den Milzknötchen.

Ratte: Sowohl bei kontrastierten als auch in injizierten Präparaten findet man keine $\mathrm{Mz}$.

\section{Großhirn.}

Bei-allen Versuchstieren kann man nicht die geringste Spur von Mz. in der Großhirnrinde finden.

\section{Periphere Nerven.}

Meerşchweinchen: Bei den kontrastierten Tieren kommen einige ovale oder langgestreckte granulareiche $\mathrm{Mz}$. in der Bindegewebshülle des Nervus ischiadicus vor. Eine Vermehrung der Mz.-Zahl in den Präparaten aus der Versuchsreihe kann nicht mit Sicherheit konstatiert werden. Die Form der Zelle und der Gehalt an Granula sind den der kontrastierten Präparate fast gleich. 


\section{Pancreas.}

Kaninchen :- Bei Tieren der Kontrastreihe findet man einige (ca. 2 im Mittel) kleine ovale, spindelförmige oder langgestreckte Mz. um die Gefäßwand in dem interlobulären Bindegewebe. Bei injizierten Tieren wird die Vermehrung der Mz.-Zahl nicht sicher konstatiert, aber die Form der Zellen zeigt die Andeutung einer Wanderung der Zelle zur Gefäßwand. (Abb. 9).

Meerschweinchen: Etwa $3 \mathrm{Mz}$. in einem Gesichtsfeld findet man in kontrastierten Präparaten um die Gefäßwand in dem interlobulären Bindegewebe.' Sie sind rundlich oval oder spindelförmig und mit mäßig reichlichen Zellgranula versehen. Durch die wiederholte Injektion des Blutkoagulans vermehrt sich die Zahl der Mz. auf ca. 7. Die Formen der Zelle sind meist spindelförmig oder langgestreckt und die Menge der Granula hat erheblich abgenommen. Bei einigen Zellen wird nachweisbar, daß sie im Begriffe sind in die Innenschicht der Gefäßwarnd inzuwandern.

Ratte: In allen Präparaten werden keine Mz. gefunden.

\section{Nebenniere.}

Kaninchen: Es gibt keine $\mathrm{Mz}$. sowohl in kontrastierten als auch in Präparaten der Injektionsreihe.

Meerschweinchen: Inder Kapsel und dem Mark der kontrastierten Nebennieren findet man mehrere (ca 5 Zellen-im Mittel) Mz., Die Formen der Zelle, welche in der Kapsel liegen, sind meist langgestreckt und die im Mark vorhandenen sind vielgestaltig. Bei Tieren der Versuchsreihe kommen die Mz. in den betreffenden Organan etwas reichlicher (ca. 8 im Mittel) vor. Ihre Granula sind aufgequollen und haben beträchtlich abgenommen.

Ratte: In der Kapsel der konstatierten Nebennieren kann man einige rundliche oder ovale granulareiche Mz. nachweisen. Durch die wiederholte Injektion des Blutkoagulans ändern sich die Befunde der Mz. nicht bedeutend.

\section{Hoden, Nebenhoden und Samenleiter.}

Kaninchen; Bei den kontrastierten Tieren befinden sich Mz. weder im Hoden noch im Nebenhoden, aber in der Faserhaut des Samenleiters findet man einige (ca. 3 Zellen im Mittel) oval, langgestreckte oder polygonale granulareiche Mz. Bei Tieren der Versuchsreihe kommen die Mz. auch nur am Samenleiter vor; wobei sie sich an Zahl etwas vermehrt (ca. 5 Zellen), nach dem Gehalt an Granula abgenommen haben.

Meerschweinchen: In der Tunica albuginea der Hoden und der Nebenhoden sowie auch in dem Bindegewebe um den Samenlieter der kontra- 
stierten Teire findet man ovale, langgestreckte oder polygonale granulareiche Mz.

Die Zahl der Zellen beträgt in Hoden und Nebenhoden dưrchschnittlich ca. $\xi$ und in der Faserhaut des Samenleiters ca. I 2. Nach der Injektion vermehrten sie sich besonders im Samenleiter um die Gefäße in der Submucosa und der Faserhaut (ca. 27 Zellen im Mittel). Die Zellgranula sind aufgequollen und haben abgenommen.

Ratte: In allen Präparaten findet man keine Mz.

\section{Ovarium, Uterus und Eileiter.}

Meerschweinchen: In der Stroma des Ovariums (ca. 4 Zéllen) sowie in der Muskelschicht des Uterus (ca. s Zellen) und in dem Bindegewebe um den Eileiter (ca. 8 Zellen) treten ovale oder spindelförmige Mz. auf, welche mit mäßig reichlichen Granula versehen sind. Nach der Injektion ansehnlich vermehrt in der Faserhaut des Eileiters (ca. 13 Zellen). Die Form der Zellen zeigen die Andeutung der Wanderüng ins Stroma des Ovariums und in den Eileiter. Die Granulamenge nimmt etwas ab.

Ratte: In der Tunica albuginea des kontrastierten Ovariums findet man nur einige rundliche oder ovale granulaarme Mz. Bei Tieren der Versuchsteihe weicht das Aussehen der Zelle nicht deutlich von dem der kontrastierten Tieren ab. In Uterus und Eileiter werden keine Mz. gefunden.

\section{Muskel.}

Als Untersuchungsmaterial wird der Bauchmuskel zum Versuche genommen.

Kaninchen: In dem intermusculären Bindégewebe der Kontrastreihe findet man einige rundliche oder ovale granulaarme Mz. besonders um die Gefässwand. Bei Tieren der Versuchsteihe wurde keine nennenswertige Veränderung der $\mathrm{Mz}$. festgestèllt.

Meerschweinchen: Bei Tieren der Kontrastreihe befinden sich durchschnittlich s relativ große ovale, spindelförmige oder langgestreckte granulareiche $\mathrm{Mz}$. in dem intermuskulären Bindegewebe. Sie stehen mit-dem Blutgefäß in innigem Zusammenhang. Nach der Injektion vermehrt sich die Zahl der Mz. um etwa 3 in einem Gesichtsfeld. Ihre Formen sind oval, spindelförmig oder polygonal. Hin und wieder gibt es Formen der Mz., aus denen hervorgeht, daß die Zellen der Gefäßwand ”sich „zu nähern im Begriffe sind.

Aufquellung und Abnahme der Zellgranula sind nicht Abrede stellen.'

Ratte: Bei der Ratten sind die Befunde von Tieren der Kontrastreihe 
ebenso wie die von Tieren aus der Veruchsreihe fast gleich denen beim Kaninchen.

\section{Haut.}

Kaninchen: In der Bauchhaut der kontrastierten Tieren kommen mehrere (ca. 5 im Mittel) kleine, rundliche, ovale oder quadratische granulareiche Mz. um die Gefäße in der Leberhaut oder um den Haarbalg sich anhäufend vor.

Bei Tieren der Versuchsreihe findet man ca. 7 Mz., welche oval, langgestreckt oder polygonal und mit geringeren Granula versehen sind. Die Veränderung der Zellformen ist nicht bedeutend:

Meerschweinchen : Es gibt einige (ca. 3 Zellen) kleine, rundliche, oder ovale granulaarme Mz. im Corium (hauptsächlich um die Gefäße oder die Haarwurzel) und in dem subcutanen Bindegewebe der Tiere der Kontrastreihe. Nach dem Experiment zeigen sie keine auffälligen Unterschiede gegenüber den kontrastierten Präparaten.

Das Aussehen der Mz. ist ungewöhnlich ähnlich dem der Lymphozyten hinsichtlich Kernfigur und Größe des Zelleibes. In dem Unterhautgewebe liegen sie meist um die Gefäße, an Gehalt der Zellgranula stark reduziert.

Ratte : In dem Corium und dem Unterhautgewebe der Kontrastreihe findet man mehrere (8 Zellen im Mittel), ovale oder spindelige granulareiche Mz. Bei der Versuchsreihe kommen ca. 10 Mz. in einem Gesichtsfeld der Präparate vor. Sie verteilen auch im Corium und in dem subcutanem Bindegewebe, entlang des Gefäßes oder Haarbalges. Die Zellgranula haben beträchtlich abgenommen und sind auch etwas aufgequollen.

\section{Erörterung und Zusammenfassung.}

Die vorstehend beschriebenen Befunde sind kurz in Tabelle 4 zusammengestellt. Es sind also zunächst drei Veränderungen der Mz., welche durch wiederholte Injektion des Blutkoagulans zustande gekommen sind, festzuhalten.

I) Die Vermehrung der $\mathrm{M} z$.-Zahl.

Wie aus der Tabelle 4 hervorgeht, ist eine Vermehrung der Mz.-Zahl am deutlichsten bei den Meerschweinchen bzw. bei deren Gefäßsystem, Magen, Dünndarm, Leber, Lunge, Harnblase und Ductus defferens aufs deutlichste nachzuweisen. Der große Unterschied in der Mz.-Zahl zwischen den kontrastierten und den injizierten Organpräparaten weißt darauf hin, daß die betreffende Veränderung nach der Injektion des Blutkoagulans nicht auf einem zufälligen Verteilungsunterschied der Mż. beruht. 
Tabelle 4.

\begin{tabular}{|c|c|c|c|c|c|c|c|c|c|}
\hline \multirow[b]{2}{*}{ Organe } & \multicolumn{3}{|c|}{ Kaninchen } & \multicolumn{3}{|c|}{ Meerschweinchen } & \multicolumn{3}{|c|}{ Ratte } \\
\hline & 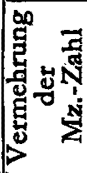 & 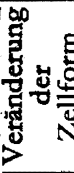 & 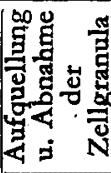 & 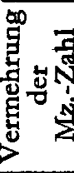 & 造 & 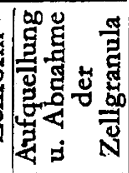 & 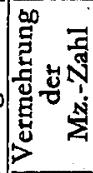 & 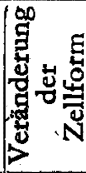 & 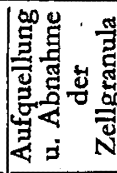 \\
\hline Zunge & - & + & + & + & + & + & - & + & + \\
\hline Oesophagus & $?$ & $?$ & $?$ & - & + & + & ? & $?$ & + \\
\hline Magen & + & + & + & + & + & + & + & + & + \\
\hline Dünndarm & 3 & ? & ? & + & + & + & ? & ? & ? \\
\hline Leber & + & + & + & + & + & + & $?$ & ? & $?$ \\
\hline Trachea & - & - & - & & & & & & . \\
\hline Zunge & + & + & + & + & + & + & + & + & 1. + \\
\hline Niere & - & - & - & - & - & - & - & - & - \\
\hline Ureter & & & & + & + & + & & & \\
\hline Harnblase & + & + & + & + & + & + & + & + & + \\
\hline $\begin{array}{l}\text { Herz u. große } \\
\text { Gefäße }\end{array}$ & $?$ & $?$ & + & + & + & + & $?$ & $?$ & $?$ \\
\hline Milz & ? & $?$ & + & + & + & + & ? & $?$ & ? \\
\hline Gehirn & & & & & & & & & - \\
\hline Peripheres Nerven & & & & - & - & - & & & \\
\hline Pancreas & ? & + & + & + & + & + & & & \\
\hline Nebenniere & 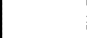 & & & + & + & + & ? & ? & ? \\
\hline $\begin{array}{c}\text { Hoden, Nebenhoden } \\
\text { u. Samenleiter }\end{array}$ & + & + & + & + & + & + & & & \\
\hline $\begin{array}{l}\text { Ovarium, Uterus u. } \\
\text {-Eileiter }\end{array}$ & & & & + & + & + & $?$ & $?$ & $?$ \\
\hline Muskel & - & - & - & + & + & + & - & - & - \\
\hline . Haut & + & \pm & + & \pm & \pm & \pm & + & + & + \\
\hline
\end{tabular}

Was die normale Verteilung der Mz. anlangt, vertreten viele Autoren die Ansicht, daß der Hauptsitz der betreffenden Zellen das lockere Bindegewebe verschiedener Organe sei und daß das perivaskuläre Bindegewebe dabei besonders hervorzugt wird (Nagayo u. Nakajima ). Miura hat in seiner ausgedehnten morphologischen Untersuchung über diese Zellen berichtet, daß die Gewebemastzellen sich in verschiedene Organe ausbreitend verteilen, reichlich in dem Bindegewebe, und mit Vorliebe um die Gefässe 
oder den Ausführungsgang der Drüsen sich anhäufend liegen.' Die erhebliche Vermehrung der $\mathrm{Mz}$. in dem 'Gefäßsystem bei meinem Versuche und die oben erwähnte normale Verteilung der $\mathrm{Mz}$. weisen zusammen darauf hin, $\mathrm{da} ß$ die $\mathrm{Mz}$. mit der Hemmung der Blutgerinnung im innigen Zusammenhang stehen müssen.

Hier taucht eine neue Frage vor uns auf, welche in der Erörterung der normalen Verteilung der $\mathrm{Mz}$. in gewißen Tietorganismen eingeschlossen wird. Es ist dies der Umstand, daß in einigen Organe die Mz. reichlich ganz unabhängig von der Anwesenheit des Blutgefäßes vorkommen. Wirklich bei der Katzenzunge oder dem Hunddarme findet man solche mit Granula förmlich vollgerüttelte $\mathrm{Mz}$. in der Submucosa oder Muskelschichte unabhängig von dem Gefäße liegen. Diese Tatsache deutet an, daß die Mz. nicht nur zur Hemmung der Blutkoagulation da sind, sondern auch noch eine andere Rolle spielen, welche sie nach ihrer normalen Verteilung in den Tierorganen sowie nach ihrem Verhalten bei gesteigertem, Stoffwechsel, wahrscheinlich als Ueberträger von Vitaminen in Frage kommen läßt. Ueber die funktionelle Bedeutung dieser Zellart also muss man weiter hin noch genauere Untersuchungen ausführen.

2) Die Veränderung der Zellform.

Wie schon oben erwähnt, ist die Form der Mz. in verschiedenen Organen rundlich, oval, spindelförmig, dreieckig, quadratisch stemförmig, langgestreckt oder sehr haüfig mit langen Ausläufern versehen, um es kurz zu sagen, die Form der Mz. ist außerordentlich mannigfaltig. Aus der vorliegenden Beschreibung der Befunde ist es möglich anzunehmen, daß bei den Kontrasttieren die meisten Zellen gewöhnlich rundlich bis spindelig sind und $\mathrm{daB}$ die langgestreckten oder polygonalen Formen selten auftreten. Aber nach der wiederohlten Inje ktion von ,,Thrombogen" kommen langgestreckte oder mit Ausläufern versehene $\mathrm{Mz}$. an Stelle der rundlichen, ovalen oder spindeligen Zellen vor.

Miura hat in seiner Abhandlung zwei Gruppen der Mz. nach der Form unterschieden. $\mathrm{Zu}$ seiner sogenannten histiozytären. Mz. (Hundeform) gehört solche Zellen, welche spindel:, oval, oder polygonal und nicht allzu selten mit einem gelappten Kerne verschen ist. Sie zeigt die Neigung Ausläufer auszustrecken. Die andere Gruppe enthält dagegen rundliche, ovale oder spindelige Mz. mit fast gleich gestaltigem Kerne, welcher meist chromatinreich ist und bisweilen eine Raḍ kernfigur zeigt. Sie gehört nach Mi u r a zu den lymphozytäre Mastzellen (Menschenform) und hat gerìngere Neigung Ausläufer auszustrecken. Er betonte, daß die histịozytäre Mz. hauptsächlich bei Hund, Kaninchen, Maus, Hühnern, Frosch und Wassermolchen vorkommt, dagegen tritt die lymphozytäre Mz. bei Menschen, Schlangen, 
Schildkröte und Bufo vulgaris auf. Aus der vorstehend erwähnten Beschreibung der Befunde scheint es mir möglich mit Recht zu sagen, daß die Zellform in Präparaten der Kontrastreihe der Menschenform und dieselbe Prâparaten der Versuchsreihe der Hundeform nach Miura genauestens entsprechen. Also kann man eine stationäre und funktionelle Form der Ḿz. unterscheiden. In der Tat möchte ich die manigfaltigen Formen und Größen der Mz. in einem Tiere eher verschiedenen physiologischen Zuständen als einer verschiedenen Entstehung der betreffenden Zellen zuschreiben.

3) Die Aufquellung und Abnahme der Zellgranula.

Die Größe und Menge der Mz.-Granula sind verschieden je nach der Art des Tieres. Nagayao und Nakajima betonten, daß die Mz.-Granula bei Ratten und Mäüsen relativ groß und tiefgefärbt, dagegen bei Katzen und Kaninchen mehr fein und schwachgefärbt seien, während diejenigen beim Hunde und Meerschweinchen eine Mittelstellung annehmen. Wie schon bekannt, ist die Widerstandfähigkeit der Mz.-Granula gegen Wasser je nach der Tierart erheblich verschieden und bei der Auslaugung der schwach widerstanḍ̣ähigen Mz.-Granula quellen einzelne Granula zuerst auf, ihre Volumina vergrößernd, danach konfluieren alle Granula miteinander im Protoplasma und werden dann in den sogenannten metachromatischen Höfen aus den Zellen ausgewaschen.

Deswegen muß man bei der Untersuchung der Mz.-Granula die geeignetste Methode vorsichtig auswählen.

In meiner früheren Mitteilung über die Darstellungsmethode der Gewebemastzellen habe ich eine neue Fixierungemethode angegeben, welche sogar die höchst löslichen Mz.-Granula relativ gut ethalten kann. Bei Mz.-Präparaten welche mit dieser Methodehergestelltwerden, kann ich keinen ansehnlichen Unterschied der Größe der Granula je nach Tierart in den Kontrastpräpataten wahrnehmen. Jẹdoch nach der Injektion von Blutkoagulans tritt die Vergrößerung der Granula sowie auch die Abnahme jhrer Menge in der Mz. der Organe von Tieren aus der Versuchsreihè deutlich zutage. Die Konturen der Zellgranula sind deutlich distinkter in Kontrastpräparaten als in Präparaten der Versuchsreihe. Die Farbennuance der Zellgranula ist auch tiefer bei den ersteren als bei den letzteren. Aus dieser Tatsache geht es hervor, daß die Größe und die Menge der Mz.-Granula je nach dem funktionellen Zustand derselben etheblich verschieden sein kann.

4) Zussamenfaßung.

In der vorstehend mitgeteilten. Arbeit werden einige Untersuchungen über die Funktion der Mz. erörtert. Die Resultate lassen sich kurz in folgende Punkte zusammenfassen.

I) An einigen Tieren, in welchen man relativ spärliche $\mathrm{Mz}$. nachweisen 
kann, wird der Versuch über das Verhalten der Mz. gegenüber dem künstlich hervorgerufenen Zustand einer erheblich beschleunigten Blutgerinnungszeit ausgeführt.

II) Durch wiederholte Injektionen fon Blutkoagulans läßt sich zuerst eine Vermehrung der Mz.-Zahl in den meisten Organen sichtlich nachweisen. Sie wird deutlich am Herz, an den großen Gefäße, añ der Lunge und am Darm wahrgenommen.

III) Die Form der Mz. ist vor und nach dem Experiment verschieden. Vor dem Versuche ist die Form meist rundlich bis spindelig, jedoch nach der Injektion des Blutkoagulans kommen häufiger langgestreckte oder mit dem Ausläufer versehene $\mathrm{Mz}$. vor. Dieses mag eine funktionelle Form und jenes eine stationäre Form der Mz. sein.

IV) Durch die Injektion von Blutkoagulans nehmen die Mz.-Granula bei fast allen Organen ab und gleichzeitig läßt sich eine Aufquellung der Granula nicht allzu selten nachweisen.

V) Aus oben erwähnten Befunden kommt Verfasser zu dem Schluß, daß die Mz. mit der Hemmung der Blutkoagulation in innigem Zusammenhang stehen durch Absonderung einer dem Heparin ähnlichen Substanz.

VI) Doch gibt es noch einige Fragen über die funktioñelle Bedeutung der Mz., wenn man die Tatsache berücksichtigt, daß sie ganz unabhängig von den Blutgefäßen in Organen auftreten oder reichlich an der Stelle vorkommen, wo der Stoffwechsel erheblich gesteigert ist.

VII) Die Form der Mz. und die Grösse der Zellgranula sind nicht wesentlich verschieden, sondern können sich je nach dem funktionellen Zustand der Mz. verändem.

Schliesslich sei es mir gestattet, meinem hochverehrten Lehrer Herm Prof. Dr. K. Okamoto meinen wärmsten Dank für seinen wertvollen Rat und die Hilfe bei der Anfertigung dieser Arbeit auszusprechen.

\section{Literatur.}

1) Asplund Borell u. Holmgren; Z. mikr. -anat. Forsch. 46: 1939.

2) Ehrlich, P.; Arch. mikrosk. Anat. 13. 1877.

3) Haser; Z. mikrosk. anat. Forsch. 41. 1937.

4) Holmgren; Anat. Anz. (Erg. Hf.) 85. 1938.

5) Holmgren u. Wilander; Z. mikr. anat. Forsch. 42. 1937.

6) Holmgren; Z. wissensch. Mikrosk. 55. 1938.

7) Holmgren; Z. mikr. -anat. Forsch. 47. 1940.

8) Is hida, D; Folia anat. jap. I1. 1938.

9) Jorpes, Holmgren u. Wilander; Z. mikr. anat. Forsch. 42. 1937.

10) Lehner; Ergeb. Anat. 25. 1924.

II) Maximow; Anat. Anz. (Erg. Hf.) 27. 1906.

12) Nagayo; Zbl. path. 43. 1928. 
Beitrag zur Erforschung der funktionellen Bedeutung der Gewebsmastzellen.

13) Nakajima; Journ. exp. med. I2. 1928.

14) Miura; Tohoku Igaku Za. 14. 1932.

I5) Rzudnitz; Arch. mikr. Anat. 22. 1883.

16) Weill; Arch. mikr. Anat. 93. I920. 


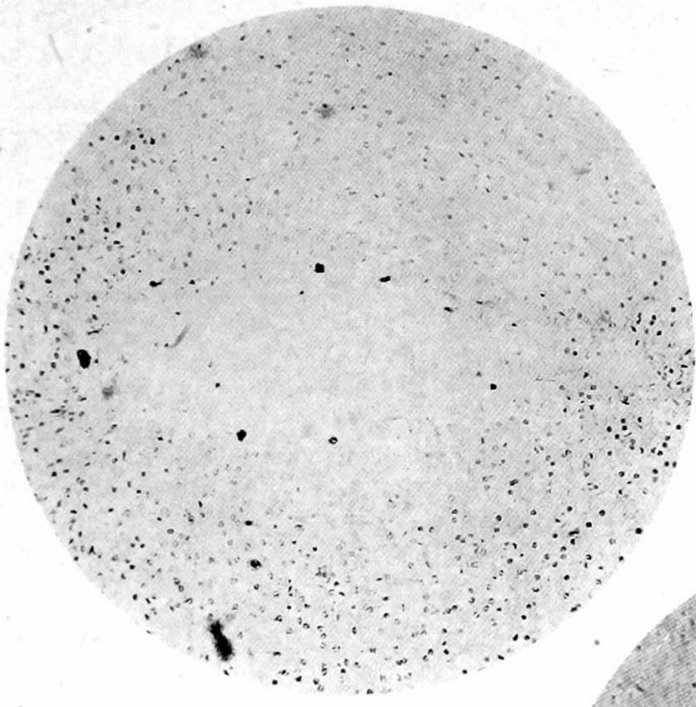

Abb. I.

Abb. 2. Mastzellen in Meerschweinchenleber. Versuchsreihe. Die Form der Zellen langgestreckt oder polygonal, ihre Granula vermindert. Mastzellenzahl nimmt zu. Fixierung und Färbung in I\% iger Trypaflavinlösung. Vergrößerung $\mathrm{I} 30 \mathrm{fach}$.

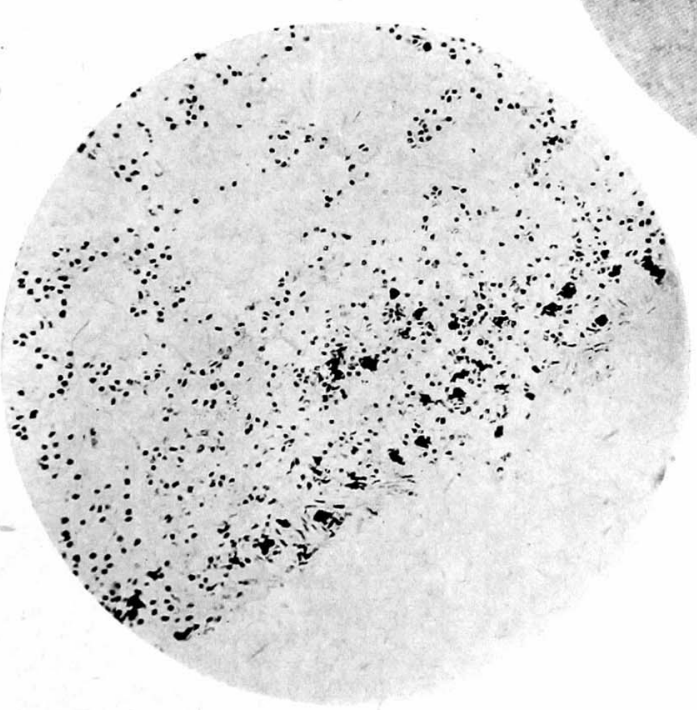

Abb. 3. Mastzellen in Meerschweinchenlunge. Kontrastreihe. Zell- und Granulakontur distinkt. Fixierung und Färbung mit I\% iger Trypaflavin-Lösung. Vergrößerung Izo fach.
Abb. r. Mastzellen in Meerschweinchenleber. Kontrastreihe. Die Form der Zellen oval, spindelig oder quadratförmig. Granulareich. Fixierung und Färbung mit I\% iger Trypaflavinlösung. Vergrößerung I 30 fach.

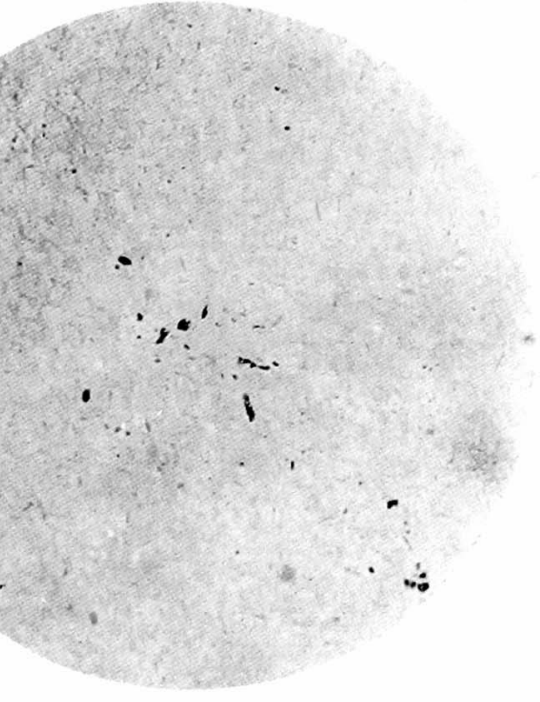

Abb. 2.

\section{Y. Kitayama Abb. 3.}



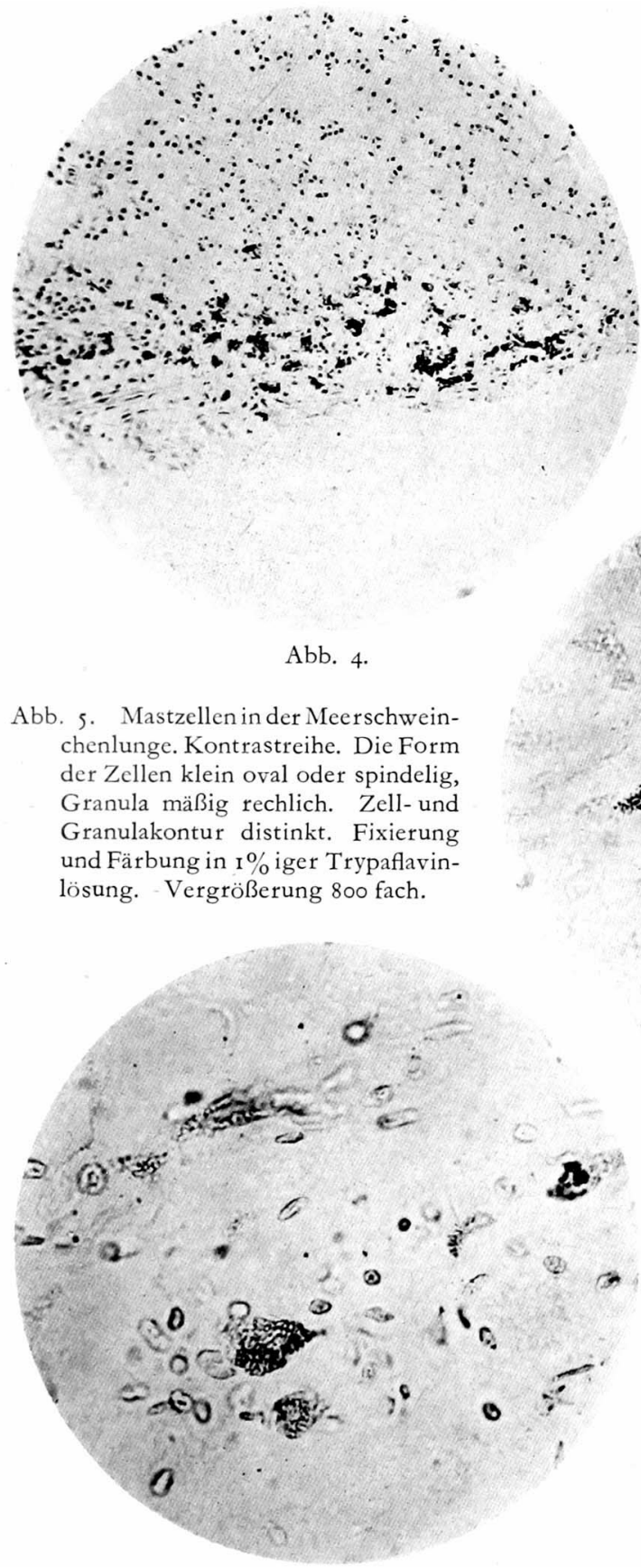

Y. Kitayama

Abb. 6.

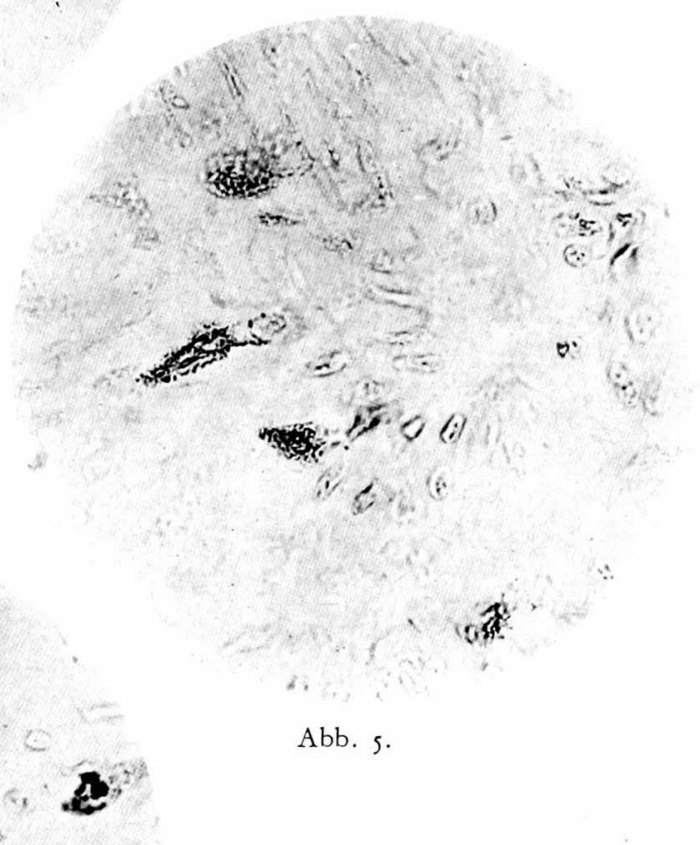

Abb. 4. Mastzellen in Meerschweinchenlunge. Versuchsreihe. Granula aufgequollen und etwas vermindert, Zahl der Mastzellen nimmt zu. Fixierung und Färbung in I\% iger Trypaflavinlösung. Vergrößerung I30 fach.

Abb. 6. Mastzellen in Mecrschweinchenlunge. Versuchsreihe. Granula aufgequollen und vermindert. Die Zellform ist langgestreckt oder polygonal. Fixicrung und Fäbung in I \% iger Trypaflavinlösung. Vergrößerung 800 fach. 


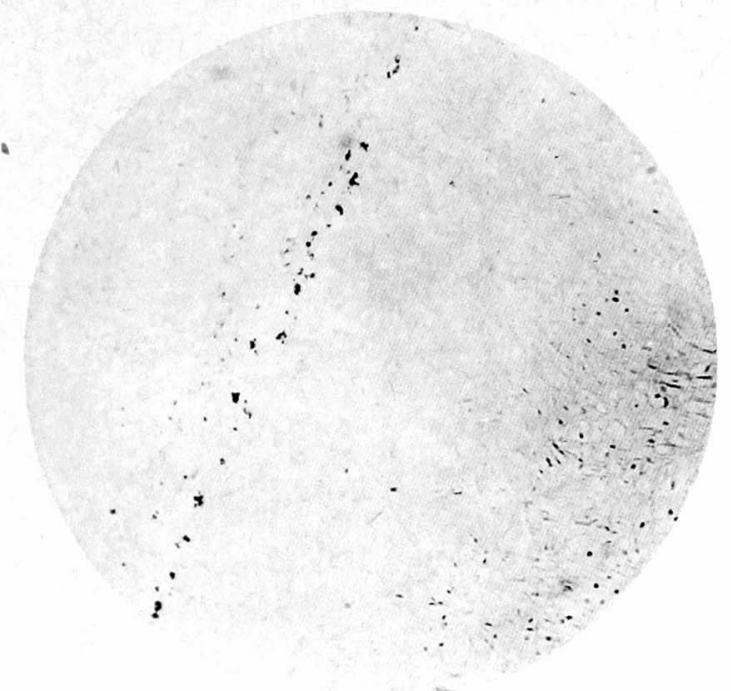

Abb. 7 .
Abb. 7. Mastzellen in Vena cava caudalis der Meerschweinchen. Kontrastreihe Zell- und Granulakontur distinkt. Die Form der Zellen oval, spindelig oder quadratförmig. Fixierung und Färbung in $1 \%$ iger Trypaflavinlösung Verg rößernng I 30 fach.

Abb. 8. Mastzellen in Vena cava caudalis Meerschweinchen. Versuchsreihe. Granula aufgequollen. Mastzellenzahl vermehrt. Fixierung und Färbung in r \% iger Trypaflavinlösung. Vergrößerung i 30 fach.

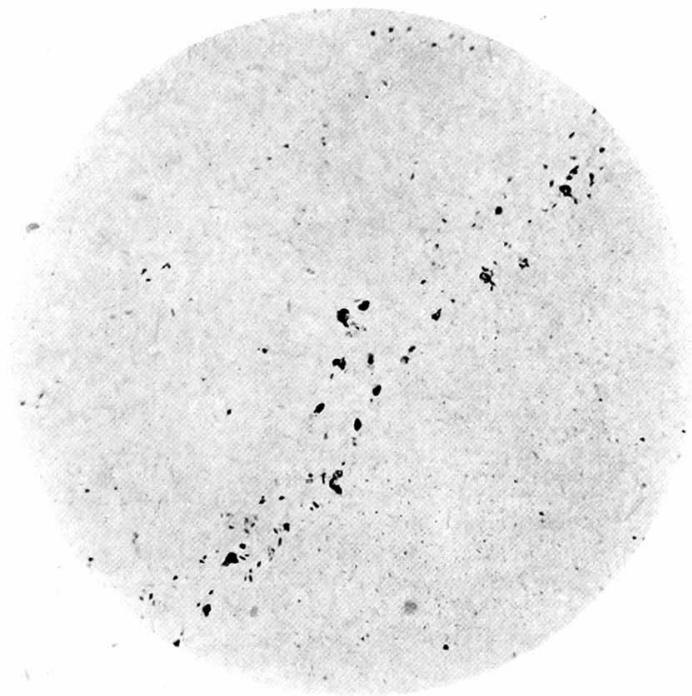

Abb. 8. 


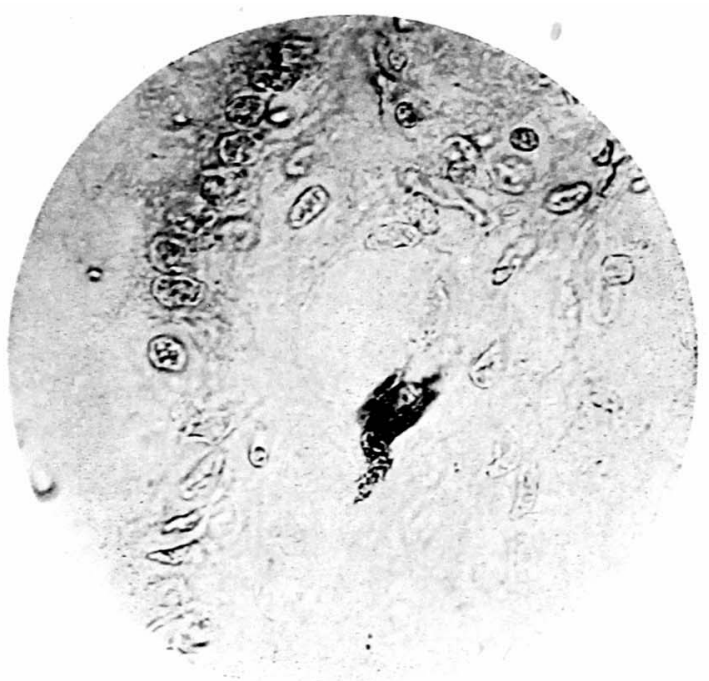

Abb. 9. Mastzellen in der KaninchenPancreas. Versuchsreihe, Langgestreckte Mastzellen mit ovalem Kerne an der Kapillarenwand. Es scheint, als ob sie im Begriff stehen sich der Kapillarenwand zu nähern. Fixierung und Färbung in $\mathrm{r} \%$ iger Trypaflavinlösung. Vergrößerung 800 fach.

Abb. 9.

Abb. ro. Mastzellen in der MeerschweinchenPancreas. Versuchsreihe. Granula noch mäßig reichlich, doch aufgequollen. Die Größe der Granula ist fast gleich der in KaninchenPancreas gesehenen. Fixierung und Färbung in $\mathrm{I} \%$ iger Trypaflavinlösung. Vergrößerung 800 fach.

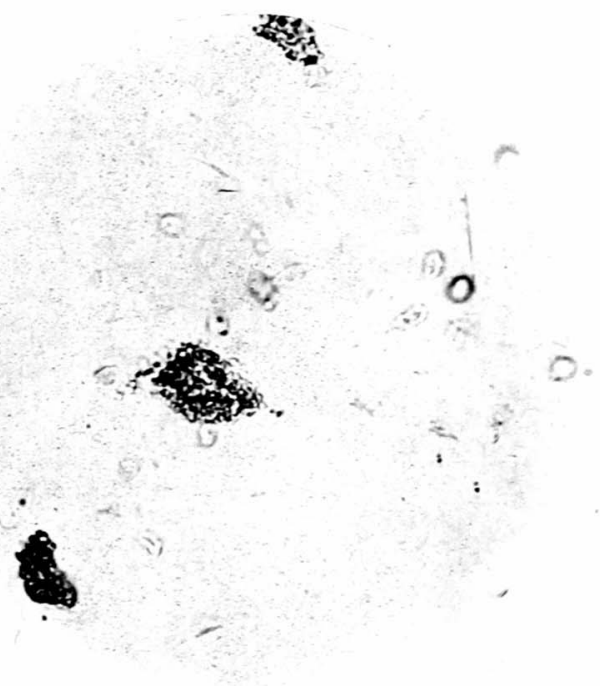

